\title{
Qualidade de vida na velhice: envelhecimento ativo e sexualidade
}

Quality of life in old age: active aging and sexuality

\section{Rosana Josso Tomazini ${ }^{1}$}

Resumo: $O$ envelhecimento populacional é tido como uma das grandes conquistas sociais do nosso tempo, mas também como o maior desafio do século XXI. Com os avanços sociais, as novas tecnologias e o progresso da medicina, a população idosa cresceu de forma exponencial. Portanto, face a esse crescimento, se faz necessário aprofundar os conhecimentos acerca do processo de envelhecimento e seus diferentes enfoques. O presente artigo resulta da revisão bibliográfica realizada em Portugal no ano de 2018. Sendo assim, busca-se compreender o conceito de Envelhecimento Ativo, refletir sobre o potencial de desenvolvimento ao longo da vida pela ótica do paradigma Lifespan, conhecer os fatores que levam as pessoas que moram na Zona Azul da Sardenha a viverem mais, e ampliar o olhar acerca da sexualidade do idoso e formas mais criativas de se relacionar.

Palavras-chave:Idosos; Envelhecimento ativo; Sexualidade do idoso.
Abstract: Population aging is seen as one of the great social achievements of our time, but also as the greatest challenge of the 21 st century. With the social advances, the new technologies and the progress of medicine, the elderly population grew exponentially. Therefore, in view of this growth, it became necessary to deepen the knowledge about the aging process and its different approaches. This article is the result of the bibliographical review held in Portugal in the year 2018 Therefore, it seeks to understand the concept of Active Aging, to reflect on the potential for lifelong development from the perspective of Lifespan paradigm, to know the factors that lead people living in the Blue Zone of Sardinia to live longer and broaden the view on the sexuality of the elderly and more creative ways of relating.

Keywords: Elderly; Active aging; Sexuality of the elderly. 


\section{Introdução}

Nas últimas décadas, o envelhecimento da população vem crescendo de forma acelerada e se tornou um fenômeno global. Em 2000, havia 600 milhões de pessoas acima de 60 anos, hoje há uma previsão de que, em 2025, este número aumente para 1.2 bilhões e em 2050 esta população chegue a 2 bilhões (Fundo de População das Nações Unidas, 2012). Segundo o IBGE, Instituto Brasileiro de Geografia e Estatística, a população brasileira manteve a tendência de envelhecimento dos últimos anos e ganhou 4,8 milhões de idosos desde 2012, superando a marca dos 30,2 milhões em 2017. 0s 4,8 milhões de novos idosos em cinco anos correspondem a um crescimento de 18\% desse grupo etário e vem se tornando cada vez mais representativo no Brasil. As mulheres são maioria expressiva nesse grupo, com 16,9 milhões (56\% dos idosos), enquanto os homens idosos são 13,3 milhões (44\% do grupo) (Paradella, 2018). Conforme a Organização Mundial da Saúde, esse fenômeno não ocorre apenas nos países ditos desenvolvidos, mas também nos países em desenvolvimento, resultando numa maior proporção de adultos idosos com 60 anos ou mais e, particularmente, adultos com 80 anos ou mais (World Health Organization, 2002, 2011).

Nessa perspectiva, o Marco Político do Envelhecimento Ativo tem 0 objetivo de criar uma nova abordagem ideológica ao envelhecimento, visando maior qualidade de vida para a população idosa (World Health Organization, 2002). 0 paradigma do Envelhecimento Ativo contempla diretrizes a serem adotadas individualmente, convidando o idoso a se responsabilizar pelo seu próprio processo de envelhecimento, e diretrizes coletivas, aquelas a serem implementadas por órgãos governamentais e comunitários, com vistas à criação de políticas públicas e demais ações que permitam o pensar coletivo.

Quando refletimos sobre o processo de envelhecimento do indivíduo, foco deste estudo, não podemos deixar de mencionar que os déficits das habilidades, comumente associados a esta fase da vida, estão apenas vagamente relacionadas com a idade cronológica das pessoas. Portanto, não existe um idoso típico. No envelhecimento, as capacidades e necessidades dos idosos não são aleatórias, e sim consequência de acontecimentos experienciados ao longo do ciclo vital, sobretudo do estilo de vida, visto que os fatores biológicos não asseguram a expressão do desenvolvimento do idoso (Firmino, Simões, \& (erejeira, 2016).

Nesse contexto, buscou-se, através do presente estudo, ampliar a visão a respeito da abordagem ideológica do Envelhecimento Ativo e entender as influências de seus conceitos. Além disso, procurou-se refletir sobre o potencial de desenvolvimento na idade avançada, baseado na Teoria Lifespan, concebida por um dos maiores e mais influentes psicólogos da atualidade, Paul Baltes. Outro fator mobilizador desse estudo foi conhecer a pesquisa realizada com os adultos longevos da Sardenha e observar quais as razões para a longevidade dessa população. Outrossim, foi de igual importância, abordar a questão da sexualidade na vida dos adultos maduros, visto que este tema está diretamente ligado ao equilíbrio emocional e à qualidade de vida desta população.

\section{Envelhecimento ativo}

Frenteà chamada "Revolução da Longevidade" (Kalache, 2013) e baseada nos Princípios das Nações Unidas para o Idoso (Ministério Público Portugal, 1991), o Marco do Envelhecimento Ativo, conceito desenvolvido e definido pela World Health Organization (2002) como o processo de otimização de oportunidades para a saúde, participação e segurança, no sentido de aumentar a qualidade de vida durante 0 envelhecimento.

Paúl e Ribeiro $(2018$, p. 3) postularam que

0 modelo depende de alguns fatores denominados "determinantes", os quais são de ordem pessoal (fatores biológicos, genéticos e psicológicos), comportamental (estilo de vida saudável e participação de vida ativa no cuidado da própria saúde), econômico (rendimentos, proteção social, oportunidades de trabalho digno), do meio físico (acessibilidade a serviç̧os de transporte, moradia evizinhança segura e apropriada, água limpa, ar puro e alimentos seguros) e, ainda, serviços sociais e de saúde (orientados para promoção da saúde e prevenção de doenças, acessíveis e de qualidade). Este grupo é complementado por dois determinantes transversais - 0 gênero e a cultura.

Cada um dos determinantes citados anteriormente resultou em atitudes e comportamentos que dependem da consciência e responsabilidade de cada indivíduo, no que diz respeito ao seu próprio processo de envelhecer, outras são direcionadas aos órgãos governamentais e organizações sociais, com 0 objetivo de criarem políticas públicas e ações voltadas para as populações, dependendo das condiç̧êes socioeconômicas de cada país. Contudo, devem ser estruturadas nas necessidades dos idosos.

Énecessário considerar que, conforme, descrito pela World Health Organization (2002), a nível individual, o Envelhecimento Ativo deve ser fomentado através de ações determinadas por processos de auto regulação emocional e motivacional. A este respeito, os conceitos a seguir devem ser observados: a autonomia, considerada no sentido do controle individual sobre a vida e a capacidade de decisão; a independência nas atividades da vida diária e nas atividades instrumentais do dia a dia, ou seja, a capacidade de cuidar de si próprio, na manutenção básica do seu corpo e no exercício de competências de manipulação do mundo externo; a expectativa de vida saudável, traduzida no tempo de vida que se pode esperar viver sem precisar de cuidados especiais; $e$ a qualidade de vida, que incorpora a saúde física, o estado psicológico, o nível de dependência, as relações sociais, as crenças pessoais e as características de ambiente em que a pessoa se encontra inserida.

Paúl \& Ribeiro (2018, p. 5-6) apontam que

Mediante esta abordagem e frente aos diversos aspectos que caracterizam o quotidiano dos mais velhos, faz-se necessário ações em termos de quatro pilares fundamentais. 0 primeiro remete ao fator saúde, que se baseia em diagnósticos médicos. 0 segundo, à segurança, ligada ao planejamento urbano e aos lugares habitados, além dos espaços privados e clima social de não violência das comunidades. 0 terceiro pilaréo da participação social na comunidade, marcada pelas relações estabelecidas com a família, os grupos de pares e o exercício da cidadania, bem como a participação ativa nesses contextos. 0 quarto pilar, incluído mais 
tarde, diz respeito ao aprendizado ao longo da vida, devido ao envelhecimento contínuo da população e a constatação da discrepância entre o tempo de formação e as exigências do mercado.

0 Marco do Envelhecimento Ativo não é considerado um documento concluído, mas um conjunto de ideias em aberto, a serem atualizadas, não obstante, continuam sendo relevantes e servem de instrumento basal para inúmeras agendas científicas. Algumas contribuições importantes foram relatadas, entre elas a pesquisa realizada em Portugal, por Paúl, Teixeira, e Ribeiro (2017). Os autores apontam para a necessidade de ampliar as dimensões propostas no paradigma do Envelhecimento Ativo e enfatizam a necessidade de introduzir uma perspectiva psicológica e levar em consideração a atitude proativa das pessoas. Ao questionar a visão determinista do modelo da OMS, o estudo analisou a operacionalidade do modelo do Envelhecimento Ativo nos fatores a seguir: saúde, componentes psicológicos, desempenho cognitivo, relações sociais, componentes biológicos e personalidade. A respeito dos aspectos psicológicos identificados na pesquisa, os autores mencionam que os indivíduos de faixa etária mais avançada valorizam de maneira particular a ausência de sofrimento psicológico, presença de felicidade e otimismo, baixo neuroticismo, boa qualidade de vida e baixa solidão, aspectos esses que, se considerados, podem permitir um envolvimento ativo com a vida (Paúl et al., 2017). Nesse sentido, os pesquisadores concluíram que esses achados reforçam a importância do funcionamento psicológico no Envelhecimento Ativo e a necessidade de novas pesquisas sobre características psicológicas específicas subjacentes ao significado subjetivo do envelhecimento ativo em idades mais avançadas (Paúl et al., 2017).

\section{Lifespan}

Ao longo do tempo, o envelhecimento foi visto, pela psicologia do desenvolvimento, como uma fase da vida em que o indivíduo amargava 0 declínio cognitivo e fisiológico, com consequente perda de produtividade, maior dependência e isolamento social. Esses conceitos não compreendiam a relação entre envelhecimento e desenvolvimento. Contudo, pesquisas como a do psicólogo alemão Paul Baltes, evidenciaram a heterogeneidade vivenciada pelos adultos maduros, observando que os alicerces do envelhecimento eram construídos de maneiras distintas, e que poderiam ser modificados de acordo com a disponibilidade do indivíduo em criar novos hábitos e desenvolver novas habilidades (Baltes, 1987; Baltes \& Baltes, 1990; Baltes \& Lindenberger, 1997).

Considerando essa dinâmica, enfatizamos a Teoria do Desenvolvimento ao Longo da Vida, conhecida como paradigma Lifespan para ilustrar esse pressuposto. 0 paradigma Lifespan foi criado por Baltes e Lindenberger (1997), e preconiza que o ser humano pode se desenvolver ao longo da vida. Os autores compreendem o desenvolvimento como um processo contínuo, multidimensional e multidirecional de mudanças orquestradas por influências genético biológicas e sócio culturais.

0 paradigma Lifespan identifica três classes de influências sobre 0 desenvolvimento: as influências ocasionadas pela idade, pela história de vida da pessoa e as influências não normativas, aquelas que podem acontecer inesperadamente (Baltes \& Lindenberger, 1997). As influências graduadas por idade se traduzem em eventos que podem ocorrer numa mesma época devido a interação dos indivíduos com o meio ambiente. Presume aquisição de papéis e competências que dependem da idade, do apoio social e dos recursos de personalidade de cada um (Neri, 2013).

As influências graduadas por história são as mudanças psicossociais que acontecem no momento da socialização e afetam de forma características os indivíduos nascidos em um mesmo tempo histórico (coorte), compreendem eventos macrossociais experienciados ao mesmo tempo por todos os componentes independentes de gênero, classe social ou raça (Neri, 2013).

As influências não normativas são eventos inesperados, a época de ocorrência é imprevisível, razão pela qual dependem dos recursos pessoais do indivíduo e não dependem do tempo histórico; geram incertezas e desafios, assim como uma sobrecarga estressora (Neri, 2013). Portanto, o desenvolvimento consiste numa ocorrência conjunta de perdas e ganhos, e na capacidade adaptativa no envelhecimento, assim como na plasticidade inteligente.

Frente ao exposto, onde o idoso desenvolve a capacidade de se adaptar aos eventos da vida através das perdas e ganhos, Baltes e Baltes (1990) conceberam a Teoria de Seleção, Otimização e Compensação (SOC). Esse modelo tem por objetivo descrever o desenvolvimento e entender como os indivíduos podem manejar as mudanças biopsicossociais que se constituem como impedimento nas trajetórias do seu desenvolvimento, em busca de um envelhecimento bem-sucedido (Neri, 2006). 0 modelo SOC é muito importante no estudo das relações entre o desenvolvimento humano, o envelhecimento e a capacidade adaptativa (Baltes \& Baltes, 1990).

No modelo SOC, a seleção determina os objetivos e resultados desejáveis, compatíveis com os recursos disponíveis. A otimização está vinculada à aquisição, aplicação e manutenção de recursos internos e externos, e ao aperfeiçoamento de meios e de recursos úteis para se atingir níveis elevados de funcionamento. Pode ser realizada mediante educação, através de treinos sistemáticos e apoio social dirigido à cognição, à saúde, à capacidade atlética e às habilidades. A compensação consiste na produção de alternativas para manter o funcionamento. São exemplos de compensação o uso de aparelhos auditivos, cadeiras de rodas, agendas para ajudar na memória e demais recursos similares (Neri, 2013). Durante toda a vida ocorre atuação sistêmica desses três mecanismos na produção do desenvolvimento e do envelhecimento bem-sucedido ou adaptativo (Baltes, 1987).

É plausível, então, considerar que, ao atingirem estados de crescente vulnerabilidade, as pessoas idosas recorram a meios externos (humanos, materiais e institucionais), no sentido de compensar essa vulnerabilidade e, ao fazê-lo, acabem por desenvolver novos valores, novos comportamentos, novas competências, resultando daí uma capacidade adaptativa mais elevada (David, 2014). Por fim, podemos considerar que as mudanças de hábitos, à medida em que haja uma diminuição do estresse, um aumento das atividades físicas e uma maior integração social, podem afetar o indivíduo e melhorar o bem-estar físico e emocional.

\section{Zona azul}

Susan Pinker, psicóloga canadense e neurocientista social, proferiu uma conferência no programa "Fronteiras do Pensamento" de 2017, em São Paulo. Na mesma ocasião apresentou a pesquisa que realizou em Villagrande, uma aldeia distante e montanhosa na ilha da Sardenha, na Itália. A aldeia foi classificada como uma das cinco Zonas Azuis do planeta onde, segundo Pinker, há seis vezes mais centenários do que no continente italiano e dez vezes mais do 
que no restante da Europa e da América do Norte. Tal fato despertou interesse da pesquisadora e os dados levantados surpreenderam a todos.

A autora encontrou moradores que vivem na ilha a muitas gerações e uma das principais características da aldeia, além de se erguer numa encosta, é que as casas foram construídas muito perto umas das outras. Ao entrevistar os centenários, Susan Pinker descobriu que, além de ter uma alimentação mediterrânea e fazer exercícios, pois sobem e descem diariamente a encosta, outro aspecto fundamental no tocante à longevidade dos habitantes, é justamente a arquitetura da aldeia. As casas, por serem muito próximas, propiciam que os moradores saibam o que está acontecendo na vida uns dos outros (Pinker, 2017).

Assim, os filhos, sobrinhos, parentes, vizinhos e pessoas de diferentes idades ajudam uns aos outros por ocasião de algum problema de saúde, falecimento, indisposição ou mesmo quando alguém estranho entra na vila todos ficam sabendo. De outra forma, eles também se divertem no dia-a-dia, jogando cartas, conversando ou participando de alguma festividade (Pinker, 2017). Dessa forma, segundo a pesquisa, é grande a sensação de pertencimento e não fazem parte das preocupações dos habitantes o medo da solidão, dos fracassos e até mesmo das demências, pois existe apoio mútuo, uma constante interação e intensa coesão social. Observa-se assim, um forte vínculo social como preditor da longevidade e a este fenômeno, Susan Pinker denominou de "Efeito Aldeia" (Pinker, 2017).

Em um resumo sobre a conferência de Susan Pinker no evento "Fronteiras do Pensamento", foi apontado que a palestrante concluiu o seguinte:"0 contato pessoal vai liberar vários neurotransmissores, vários hormônios que aumentam a confiança entre as pessoas. E também diminui o estresse, e isso pode ser medido pelos níveis de cortisol na saliva e no sangue. E também é algo analgésico. Vocês sabiam que o contato pessoal é analgésico natural? E também induz o prazer. Dopamina é gerada quando você dá um high five e um tapinha nas costas. Ou, como vocês fazem aqui em Porto Alegre, que é dar um beijo na bochecha." (Thomé, 2017).

Thomé (2017) aponta que, atualmente, 25\% da população global declaram não ter com quem conversar. Assim, a psicóloga Susan Pinker encerrou sua conferência no "Fronteiras do Pensamento": "Em primeiro lugar, podemos planejar e construir as nossas cidades em comunidades com lugares de encontros - aqueles Terceiros Espaços, lugares em que a gente se sente à vontade. Nós podemos construir interações pessoais e incluir esses relacionamentos nos nossos sistemas de educação e de saúde, incluir isso no nosso tempo livre, na nossa vida profissional. Você pode colocar isso na sua agenda como vocêfaz com o exercício. Isso fortalece o seu sistema imunológico, libera hormônios de bem-estar na sua corrente sanguínea e no cérebro. Ajuda as crianças a floresceram e a aprenderem. Ajuda os adultos a viverem uma vida mais longa, mais feliz e mais saudável. Eu chamo isso de ' 0 efeito aldeia'. $E$ você construir e sustentar esse tipo de comunidade é uma questão de vida ou morte" (Thomé, 2017)

\section{Sexualidade assíncrona}

Se a interação social é preditora de longevidade, saúde e bem-estar, é natural que se pense a questão da sexualidade na idade avançada. No enveIhecimento, a sexualidade varia tanto quanto os demais comportamentos, mas isso não implica necessariamente uma redução drástica da resposta sexual, já que ela depende fundamentalmente da atitude que a pessoa adota diante da vida. Ocorre de maneira extremamente individual e não se processa do mesmo modo em todas as épocas, nem sequer da mesma forma em todos os indivíduos (Pascual, 2002).

Dessa forma, o exercício da sexualidade entre os casais de idosos também, avança ao longo dos anos de forma heterogênea e particular. 0 processo de envelhecimento não impede o indivíduo de ter experiências sexuais, pois não se trata de uma fase meramente assexuada (Fávero \& Barbosa, 2011).

Conforme o paradigma Lifespan, postulado por Baltes e Lindenberger (1997), o desenvolvimento ao longo da vida é um processo contínuo, multidimensional e multidirecional de mudanças, cuja plasticidade enfatiza as potencialidades de crescimento do sujeito idoso. Então, podemos pensar que as perdas físicas que caracterizam esta fase da vida não impedem que as formas de intimidade sejam flexibilizadas e que o prazer e a satisfação sejam reinventados pelo casal.

Resultados que corroboram com as proposições teóricas de Baltes (1987) foram encontrados em um estudo de caso realizado por McCarthy e Pierpaoli (2015), quando, através de um processo clínico qualitativo, um casal de idosos com queixas de impotência do homem e insatisfação da mulher, foi tratado a partir da abordagem Good Enough Sex - GES (Metz \& McCarthy, 2007).

A abordagem GES oferece um modelo integrativo e abrangente para promover o sexo vibrante e sustentador nos relacionamentos, visando melhorar a intimidade, o prazer, o erotismo, a satisfação e a coesão do casal. 0 modelo preconizado pela abordagem GES considera o estágio de envelhecimento dos parceiros e estimula o casal a olhar para o seu relacionamento sexual de forma flexível, considerando os fatores psicológicos, biológicos e sociais. Convida-os a abraçar a sexualidade como forma de energizar e promover o bem-estar relacional ao longo da vida (Metz \& McCarthy, 2010).

Os achados apresentados sugerem que as perdas físicas não impedem a flexibilidade e os ganhos eróticos de intimidade e satisfação ao longo da vida.

0 tratamento psicoterapêutico compreendeu nove sessões realizadas num período de cinco meses, incluindo três sessões de prevenção à recaída durante o período de dois anos. Uma nova forma do casal se ver foi trabalhado em sessão. Não eram mais um homem e uma mulher em busca da satisfação sexual individualizada, mas sim uma dupla, onde a felicidade sexual era responsabilidade dos dois (Metz \& McCarthy, 2007).

A partir do momento em que os dois perceberam a necessidade de se desprender do modelo antigo de relacionamento sexual, cuja expectativa era de manter o desempenho da juventude, tornaram-se amigos íntimos. 0 casal se dispôs a criar cenários sexuais diferenciados, ter experiência assíncronas, criaram ambientes distintos daqueles que estavam acostumados, exploraram 0 olfato, o toque, incluindo elementos lúdicos na relação. Dessa forma, a vivência da sexualidade foi ampliada, e não mais baseada apenas no ato sexual (Metz \& McCarthy, 2007). Vale salientar que ambos os conceitos de Baltes (1987), 0 da plasticidade, que explora a promoção das potencialidades e crescimento, assim como o da multidirecionalidade, que descreve a não linearidade de determinado domínio, foram constatados neste estudo, ressaltando a importância da adaptação para que os casais possam vivenciar de forma saudável sua sexualidade.

Ao introjetarem a abordagem GES, e estarem abertos a uma nova maneira de se relacionar, quando o sexo não flui para o intercurso, homens 
e mulheres mais velhos podem se orgulhar da possibilidade de apreciar a variabilidade de seus encontros (McCarthy \& Pierpaoli, 2015). A vivência da sexualidade na idade avançada pode ser enriquecedora, fisiologicamente possível e uma fonte importante de intimidade e carinho. É uma forma de cuidado mútuo, de estimular a cumplicidade e a realização pessoal (Urquiza, Thumala, Arnold-(athalifaud, 0jeda, \& Vogel, 2008).

\section{Discussão}

A partir dos temas abordados neste estudo, vimos que o crescimento da população idosa é um fenômeno atual. Assim, em muitos países, pesquisadores e gestores da área da saúde estão atentos para o desenvolvimento de estratégias e intervenções que mantenham a autonomia e a independência dessa população. Não basta viver mais, mas este tanto de vida a mais deve ser vivido com qualidade. Essa ideia mobilizou os esforços de inúmeros profissionais atuantes junto a OMS, e resultou em diretrizes de base do paradigma do Envelhecimento Ativo, uma nova forma de ver o envelhecimento.

Esse novo olhar apontou a interação social como um dos aspectos mais relevantes para a qualidade de vida do idoso, visto que a sensação de solidão é um preditor de sofrimento e causa de inúmeras doenças incapacitantes. Portanto, a participação social, a convivência com grupos, sejam eles familiares, amigos ou grupos específicos, são extremamente importantes para 0 bem-estar emocional e saúde mental do idoso.

Através da teoria Lifespan, podemos observar que o processo de enveIhecimento é único e depende de perdas e ganhos. Assim, entendemos que os ganhos que o indivíduo tem na infância o afetam de modo subjetivo, essa forma subjetiva de ver o mundo segue marcando-0 ao longo da vida adulta e, na velhice, da mesma forma, ele vai enfrentar as perdas de um jeito próprio.

Dessa forma, podemos dizer que o envelhecimento é um processo que se dá de forma distinta para cada pessoa, o que nos leva a inferir que, embora 0 envelhecimento fragilize os idosos, através de recursos como a seleção, otimização e compensação, é possível desenvolver novos comportamentos e atitudes em busca de maior êxito e maior satisfação com a vida.

Nesse sentido, a sexualidade é um componente fundamental da saúde geral do adulto maduro. Assim, todo o idoso pode ter momentos de intimidade, com erotismo e prazer sexual, basta estar disponível para experienciar. Contudo, devemos lembrar que a sexualidade na idade madura não éa mesma das fases anteriores do ciclo vital. Novas maneiras de demonstrar interesse devem ser abordadas de forma assíncrona. A sexualidade assíncrona vê o casal como uma dupla de aliados, cúmplices na relação, que muitas vezes, não se trata apenas do ato sexual em si, mas de todo um envolvimento na criação de cenários como, por exemplo, acender velas, colocar flores na mesa, preparar um banho de banheira. 0 esforço de criar 0 ambiente propício estimula e desperta o desejo, mesmo que a ação não leve ao ato sexual em si, ela pode gerar momentos de carinho e cuidados recíprocos.

Percebemos, assim, que o fato do idoso poder aprender novos comportamentos ao longo da vida, pode ser um facilitador de maior participação social. A exemplo dos centenários que vivem na Sardenha, saber viver em comunidades, poder cuidar e ser cuidado, pode representar uma maneira satisfatória de se viver.

\section{Considerações finais}

0 envelhecimento da população acarreta um conjunto de impactos na sociedade, sendo necessário encontrar novas respostas e novos modelos para enfrentar os desafios que se apresentam. 0 paradigma do Envelhecimento Ativo é um modelo que remete a possibilidades de ações mais justas no sentido de melhorar as consequências adversas à passagem do tempo. Contudo, está aberto a novas contribuições no que se refere à subjetividade da população idosa.

Cabe lembrar que este é um cenário variável, pois a possibilidade de desenvolvimento ao longo da vida torna o processo de envelhecimento muito mais dinâmico, sugerindo novos comportamentos e atitudes como forma de obter melhor qualidade de vida. Contrariando estereótipos, vimos que a interação social, as formas criativas de se relacionar e viver a sexualidade, contribuem para a autonomia e independência do idoso, tornando-0 um ser ativo e funcional na busca do próprio envelhecimento bem-sucedido. Por fim, sendo o envelhecimento um fenômeno universal, envelhecer com qualidade é uma conquista.

\section{Referências}

Baltes, P. (1987). Proposições teóricas da psicologia do desenvolvimento da vida: Sobre a dinâmica entre crescimento e declínio. Psicologia do Desenvolvimento, 23, 611-626.

Baltes, P., \& Baltes, M. (1990). Psychological perspectives on successful aging. The model of selective optimization with compensation. In P. Baltes, \& M. Baltes (Eds.), Successful aging: Perspectives from behavioral sciences (pp. 1-34). New York: Cambridge University Press

Baltes, P., \& Lindenberger, U. (1997). Emergence of a powerful connection between sensory and cognitive functions across the adult life span: A new window to the study of cognitive aging?. Psychology and Aging, 12(1), 12-21. doi: 10.1037/0882-7974.12.1.12

David, M. J. C. (2014). Plasticidade cognitiva e envelhecimento bem-sucedido: Otimização e compensação funcional através das atividades de vida diária instrumentais (Dissertação de mestrado). Recuperado de http://dspace. uevora.pt/rdpc/handle/10174/10928

Fávero, M. F., \& Barbosa, S. C. S. (2011). Sexualidade na velhice: Os conhecimentos e as atitudes dos profissionais de saúde. Terapia Sexual, 14(2), $11-39$.

Firmino, H., Simões, M. R., \& Cerejeira, J. (2016). Saúde mental das pessoas mais velhas. Lisboa: Lidel.

Fundo de População das Naç̃ões Unidas. (2012). Resumo executivo - Envelhecimento no século XXI: Celebração e desafio. Recuperado de https://www. unfpa.org/sites/default/files/pub-pdf/Portuguese-Exec-Summary_0.pdf

Kalache, A. (2013). The longevity revolution: Creating a society for all ages. Adeleide Thinker in Residence 2012-2013. Adeleide: Govermment of South Australia.

McCarthy, B., \& Pierpaoli, C. (2015). Sexual challenges with aging: Integrating the GES approach in an elderly couple. Journal of Sex \& Marital Therapy, 41(1), 72-82. doi: 10.1080/0092623X.2013.831004

Metz, M. E., \& McCarthy, B. W. (2007). The "Good-Enough Sex" model for couple sexual satisfaction. Sexual and Relationship Therapy, 22(3), 351362. doi: 10.1080/14681990601013492

Metz, M. E., \& McCarthy, B. W. (2010). Desejo duradouro. Nova York, NY: Routledge. 
Ministério Público Portugal. (1991). Princípios das Naçōes Unidas para as pessoas idosas. Adotados pela resolução 46/91 da Assembleia Geral das Naç̃oes Unidas, de 16 de dezembro de 1991. Recuperado de http://gddc. ministeriopublico.pt/sites/default/files/

princ-pessoasidosas.pdf

Neri, A. L. (2006). 0 legado de Paul B. Baltes à psicologia do desenvolvimento e do envelhecimento. Temas em Psicologia, 14(1), 17-34. Recuperado de http://pepsic.bvsalud.org/pdf/tp/v14n1/v14n1a05.pdf

Neri, A. L. (2013). Conceitos e teorias sobre o envelhecimento. In L. F. Malloy-Diniz, D. Fuentes, \& R. M. Cosenza (Orgs.), Neuropsicologia do envelhecimento: Uma abordagem multidimensional (pp. 17-42). Porto Alegre: Artmed.

Pascual, P. C. (2002). A sexualidade do idoso vista com um novo olhar. São Paulo, SP: Loyola.

Paradella, R. (2018, abril 26). Número de idosos cresce 18\% em 5 anos e ultrapassa 30 milhões em 2017. Agência IBGE Notícias. Recuperado de https://agenciadenoticias.ibge.gov.br/agencia-noticias/2012-agencia-de-noticias/noticias/20980-numero-de-idosos-cresce-18-em-5-anose-ultrapassa-30-milhoes-em-2017

Paúl, C., \& Ribeiro, 0. (2018). Manual de envelhecimento ativo (2a ed.). Lisboa: Lidel.

Paúl, C., Teixeira, L., \& Ribeiro, 0. (2017). Active aging in very old age and the relevance of psychological aspects. Frontiers in Medicine, 4(181), 1-7. doi: 10.3389/fmed.2017.00181

Urquiza, A., Thumala, D., Arnold-Cathalifaud, M., Ojeda, A., \& Vogel, N. (2008). Sexualidad em la tercera edad. La imagen de los jóvenes universitarios. Ponto e Virgula, 4, 358-374. Recuperado de http://repositorio.uchile.cl/ bitstream/handle/2250/121679/Sexualidad_en_la_tercera_edad.pd?? sequence $=1$ \&isAllowed $=y$

Pinker, S. (2017, Dezembro 5). O efeito aldeia [arquivo de vídeo]. Recuperado de https://www.youtube.com/watch?v=JXBE_76Ccaw

Thomé, L. (2017, Dezembro 4). Resumo - Susan Pinker. 0 efeito aldeia. Fronteiras do Pensamento. Recuperado de https://www.fronteiras.com/ resumos/o-efeito-aldeia-poa

World Health Organization. (2002). Active ageing: A policy framework. Retrieved from https://apps.who.int/iris/bitstream/handle/

10665/67215/WHO_NMH_NPH_02.8.pdf?sequence=1

World Health Organization. (2011). Global health and aging. Retrieved from https://www.who.int/ageing/publications/global_health.pdf?ua=1. 